\title{
Early detection of cardiac surgery-associated acute kidney injury by microRNA-21
}

\author{
Arvin $\mathrm{P}^{1}$, Samimagham $\mathrm{HR}^{2}$, Montazerghaem $\mathrm{H}^{3}$, Khayatian $\mathrm{M}^{4}$, Mahboobi $\mathrm{H}^{5}$, Ghadiri Soufi $\mathrm{F}^{6}$ \\ Department of Physiology, Faculty of Medicine, Hormozgan University of Medical Sciences, \\ Bandar Abbas, Iran. Dr.F.G.Soufi@hums.ac.ir
}

\begin{abstract}
We tested the hypothesis whether microRNA-21 (miR-21) can detect CSA-AKI earlier than serum creatinine (sCr). A total of 103 patients scheduled to undergo cardiac surgery. CSA-AKI was defined as $\mathrm{sCr}>0.3 \mathrm{mg} / \mathrm{dl}$ $24 \mathrm{~h}$ after surgery. The patients were divided into two groups according to whether or not developing AKI after surgery. Serum and urinary miR-21 were measured prior to, and 6, 12 and $24 \mathrm{~h}$ after surgery.

Baseline serum and urinary levels of miR-21 in AKI group were lower than in non-AKI group. Moreover, the levels of miR-21 were significantly lower $6 \mathrm{~h}$ after surgery for serum, and 6 and $12 \mathrm{~h}$ after surgery for urine samples than those before surgery in AKI group. Area under the curve (AUC) of the receiver operating characteristic (ROC) values were 0.81 (95\% Cl: 0.65-0.97) for serum miR-21 (6 h after surgery), 0.90 (95\% Cl: 0.79-0.99) for urine ( $6 \mathrm{~h}$ after surgery), and 0.86 (95\% Cl: $0.71-0.98)$ for urine (12 h after surgery).

While both postoperative serum and urinary miR-21 levels can predict AKI development, urinary miR-21 especially $6 \mathrm{~h}$ after surgery is a more reliable marker than serum miR-21 for detection of established CSA-AKI (Tab. 1, Fig. 3, Ref. 43). Text in PDF www.elis.sk.

KEY WORDS: cardiac surgery, acute kidney injury (AKI), miRNA-21.
\end{abstract}

\section{Introduction}

Cardiac surgery-associated acute kidney injury (CSA-AKI) is common and has high mortality and morbidity rates (1-4). Management of SCA-AKI is costly and often the affected individuals run the risk of death due to electrolyte imbalance or excessive toxins in plasma (5).

Early diagnosis of CSA-AKI is vital and can alter the prognosis of the diseases. It has been shown that postoperative changes in serum creatinine $(\mathrm{sCr})$ levels are not reliable markers of CSA$\mathrm{AKI}(4,6)$. SCr level usually rises in late stages of kidney injury when around $50 \%$ of the kidney function is lost (7).

${ }^{1}$ Department of Physiology, Faculty of Medicine, Hormozgan University of Medical Sciences, Bandar Abbas, Iran, ${ }^{2}$ Department of Nephrology, Hormozgan University of Medical Sciences, Bandar Abbas, Iran, ${ }^{3}$ Department of Cardiac surgery, Hormozgan University of Medical Sciences, Bandar Abbas, Iran, ${ }^{4}$ Department of Biochemistry, Faculty of Medicine, Hormozgan University of Medical Sciences, Bandar Abbas, Iran, ${ }^{5}$ Department of Internal Medicine, Infectious and Tropical Diseases Research Center, Hormozgan University of Medical Sciences, Bandar Abbas, Iran, and ${ }^{6}$ Molecular Medicine Research Center, Hormozgan University of Medical Sciences, Bandar Abbas, Iran

Address for correspondence: $\mathrm{F}$. Ghadiri Soufi, $\mathrm{PhD}$, Molecular Medicine Research Center, Hormozgan University of Medical Sciences, Jomhoury Boulevard, Bandar Abbas, Iran.

Phone/Fax: +98.76.33333012

Acknowledgments: This study was funded by the Molecular Medicine Research Center at Hormozgan University of Medical Sciences (grant number: MU-95-3).
Despite the fact that during recent years several studies have tried to introduce reliable markers such as cystatin C, kidney injury molecule-1 (Kim-1), neutrophil gelatinase-associated lipocalin (NGAL), etc. for early diagnosis or preventive treatment of CSA-AKI (8-11), no reliable marker exists for early diagnosis of CSA-AKI, and the researchers keep working on the available markers, their accuracy and prognostic value for diagnosis of CSA-AKI (12-16).

MicroRNAs are post-transcriptional regulatory factors for gene expression. Their role has been shown in signaling cascades involved in the pathogenesis of renal diseases (17). It has been proposed that a more specific biomarker, microRNA-21 (miR-21) has a role in mechanisms which are responsible in pathogenesis of AKI (18)

AKI causes inflammation as a result of damage to tubular epithelial cells (19). The mechanism of inflammation lies in the release of pro-inflammatory mediators from injured tubular epithelial cells including interleukin-6 (IL-6), IL-1 $\beta$, IL-8, tumor necrosis factor (TNF), transforming growth factor- $\beta 1$ (TGF- $\beta 1)$, and monocyte chemotactic protein-1 (MCP-1) $(18,20,21)$.

After a severe AKI, fibrosis occurs in renal proximal tubules. The role of miR-21 in the process of fibrosis after AKI has been shown is several diseases (18). Both inflammation and fibrosis have roles in the pathogenesis of AKI, and miR-21 has a complicated role in both mechanisms. The overexpression of miR-21 has been shown in inflammation and fibrosis following AKI $(18,22)$.

One important mechanism of injury in CSA-AKI is ischemia reperfusion injury (4). Some studies suggest a protective role for miR-21 during the short period after ischemia reperfusion injury 
$(4,18)$. However, it seems that an imbalance in overexpression or underexpression of miR-21 may be involved in adverse outcomes in CSA-AKI (18).

Increasing evidences are reported about the effectiveness of prophylactic usage of medications for prevention of CSA-AKI (23-25). Therefore, finding reliable markers of CSA-AKI can be used for evaluation of the effectiveness of these medications and their mechanism of action (26).

To the best of our knowledge only two studies have previously shown the role of miR-21 as a diagnostic biomarker for the detection of CSA-AKI $(27,28)$.

In this regard $\mathrm{Du}$ et al have reported higher levels of serum and urine miR-21 in CSA-AKI patients $19.5 \mathrm{~h}$ after cardiac surgery (27). Surprisingly, in another study, Gaede et al have recently reported that while the baseline (prior to surgery) serum miR-21 level was significantly lower in CSA-AKI patients, its value did not differ $4 \mathrm{~h}$ after surgery between the patients with and without CSA-AKI (28). Although both studies have reported the accuracy of miR-21 in prediction of CSA-AKI, no one has studied whether miR-21 can detect CSA-AKI before $\mathrm{sCr}$ increment.

Early detection of CSA-AKI is vital for the management of patients. Timing of renal replacement therapy in CSA-AKI is an important decision in the management of CSA-AKI in these patients. Preemptive renal replacement therapy is shown to improve the treatment results in CSA-AKI [29]. For early diagnosis and initiation of renal replacement therapy, reliable biomarkers of CSA-AKI are useful.

The aim of this study was to test the hypothesis that miR-21 can detect CSA-AKI earlier than sCr. To achieve this goal, we measured serum and urine concentrations of miR-21 in patients prior to, and 6,12 , and $24 \mathrm{~h}$ after cardiac surgery using real-time PCR.

\section{Materials and methods}

\section{Ethics Statement}

The study has been approved by the Ethical Committee of Hormozgan University of Medical Sciences, and adhered to the tenets of the Declaration of Helsinki. In addition, a written informed consent was obtained from all patients who accepted to participate in the study. Patients' information were kept confidential.

\section{Study design and patients}

Based on the prevalence of AKI (5-45\%), a total of 103 consecutive patients (age 48-70 years) scheduled to undergo elective major cardiac surgery (coronary artery bypass grafting and/or valve replacement) between June and November 2015 with the use of extracorporeal circulation (ECC) at the Shahid Mohammadi Hospital, Bandar Abbas, Iran. The clinical history and results of physical examination, laboratory tests, echocardiography and coronary angiography were assessed for all patients. Cardiac surgery was performed according to the standard clinical practice.

The patients were divided into two groups according to whether or not developing AKI after cardiac surgery. Based on Kidney Disease Improving Global Outcomes (KDIGO) criteria (30) and the aim of this study (detection of AKI within $24 \mathrm{~h}$ postoperative period), AKI was defined as an increase in $\mathrm{sCr}>0.3 \mathrm{mg} / \mathrm{dl} 24 \mathrm{~h}$ after surgery. Based on measured $\mathrm{sCr}$ of the 103 patients undergoing elective major cardiac surgery, 17 patients developed CSA-AKI. To match patients' age and sex, as well as some exclusion criteria, only 14 patients ( 7 male and 7 female) were included in AKI group. Sex and age matched patients who were showing no evidence of postoperative increase in $\mathrm{sCr}$ formed the non-AKI group $(\mathrm{n}=14)$.

\section{Inclusion and exclusion criteria}

Based on the Cleveland clinic score, all candidates for open cardiac surgery who had not the risk factors including congestive heart failure, chronic obstructive pulmonary disease, infectious diseases, end-stage renal diseases, insulin-requiring diabetes, preoperative proteinuria, emergency surgery, and angiography within recent 72 hours were included in the study. Patients who avoided to complete written informed consent, and patients who had repeated cardiac surgery due to bleeding, as well as patients who their $\mathrm{sCr}$ levels increased within 48 or $72 \mathrm{~h}$ after cardiac surgery (18 and 3 Patients, respectively) were excluded from the study.

\section{sample collection and Preparation}

Urine and venous blood samples were collected $6 \mathrm{~h}$ prior to (baseline sample) and 6, 12, 24, 48 and $72 \mathrm{~h}$ after cardiac surgery. All samples were processed within $1 \mathrm{~h}$ after collection. Urine samples were centrifuged at $4{ }^{\circ} \mathrm{C}$ at $10000 \mathrm{~g}$ for $10 \mathrm{~min}$ and serum samples were centrifuged at $3000 \mathrm{rpm}$ for $15 \mathrm{~min}$. Aliquots of the samples were added to RNase/DNase-free tubes and stored at -80 ${ }^{\circ} \mathrm{C}$ (27). SCr was determined $6 \mathrm{~h}$ prior to, and 24,48 and $72 \mathrm{~h}$ after cardiac surgery using a 912 automatic biochemistry analyzer (Hitachi, Japan), and serum and urine miR-21 were measured $6 \mathrm{~h}$ prior to, and 6, 12 and $24 \mathrm{~h}$ after cardiac surgery using real-time PCR.

\section{Real-time PCR}

Total RNA was extracted from serum and urinary samples, using the miRneasy mini kit (Qiagen, Hilden, Germany) according to manufacturer's instructions. The samples were spiked with $1 \mathrm{ng}$ of C. elegans miR-39 mimic (cel-miR-39 miScript miRNA, Qiagen), as internal control miRNA.

For each sample, total RNA $(1 \mu \mathrm{g})$ was reversely transcribed using miScript II RT cDNA synthesis Kit (Qiagen) according to the manufacturer's instructions. Each cDNA was used as a template for separate assay for miR-21 and cel-miR-39 quantitative real-time RT-PCR with miScript SYBR Green PCR Kit (Qiagen) using miScript primer assays (Qiagen) for human miR-21 and C. elegans miR-39 respectively. All reactions were performed in triplicate on a Corbett Rotor-Gene RG-6000 (Australia). The $2^{-\Delta c t}$ method, 2-(CT[miR-21] - CT[cel-miR-39]) was used to evaluate the relative expression of miR-21 (27). Group comparisons were expressed as mean $2^{-\Delta c t} \pm \mathrm{SD}$.

\section{Study statistics}

For data analysis, IBM SPSS version 24.0 software were used. Shapiro-Wilks test was used to assess the normality of distribution of data. Repeated measures ANOVA with Bonferroni post hoc test were used for detection of $\mathrm{sCr}$ and miR-21 within 
$626-631$

Tab. 1. Demographic variables in AKI and non-AKI groups.

\begin{tabular}{|c|c|c|c|}
\hline Variable & $\mathrm{AKI}(\mathrm{n}=14)$ & Non-AKI $(n=14)$ & $P$ value \\
\hline \multicolumn{4}{|l|}{ Sex, n (\%) } \\
\hline Male & $7(50 \%)$ & $8(57.1 \%)$ & 0.799 \\
\hline Female & $7(50 \%)$ & $6(42.9 \%)$ & 0.816 \\
\hline Age (year) & $58.57 \pm 12.02$ & $58.86 \pm 10.28$ & 0.974 \\
\hline Height $(\mathrm{cm})$ & $161.50 \pm 9.89$ & $162.57 \pm 9.06$ & 0.768 \\
\hline Weight (kg) & $61.21 \pm 10.63$ & $64.29 \pm 17.43$ & 0.578 \\
\hline $\mathrm{BMI}\left(\mathrm{kg} / \mathrm{m}^{2}\right)$ & $23.61 \pm 4.37$ & $24.06 \pm 4.69$ & 0.799 \\
\hline Hypertension, n (\%) & $8(57.1 \%)$ & $7(50 \%)$ & 0.705 \\
\hline Type 2 Diabetes Mellitus, n (\%) & $6(42.9 \%)$ & $5(35.7 \%)$ & 0.699 \\
\hline Duration of aorta clamp (min.) & $94.71 \pm 39.11$ & $70.35 \pm 11.92$ & 0.041 \\
\hline Duration of surgery (min.) & $141.07 \pm 40.36$ & $117.21 \pm 26.25$ & 0.075 \\
\hline Ejection fraction (\%) & $44.28 \pm 9.16$ & $43.92 \pm 12.58$ & 0.932 \\
\hline Serum Cr before surgery $(\mathrm{mg} / \mathrm{dl})$ & $0.83 \pm 0.10$ & $0.79 \pm 0.11$ & 0.107 \\
\hline Serum miR-21 before surgery $\left(2^{-\Delta \mathrm{ct}}\right)$ & $5.41 \pm 1.84$ & $8.23 \pm 1.22$ & 0.048 \\
\hline Urine miR-21 before surgery $\left(2^{-\Delta \mathrm{ct}}\right)$ & $7.73 \pm 2.26$ & $11.96 \pm 1.87$ & 0.039 \\
\hline Mortality, n (\%) & $1(7.1 \%)$ & $0(0 \%)$ & 1 \\
\hline
\end{tabular}

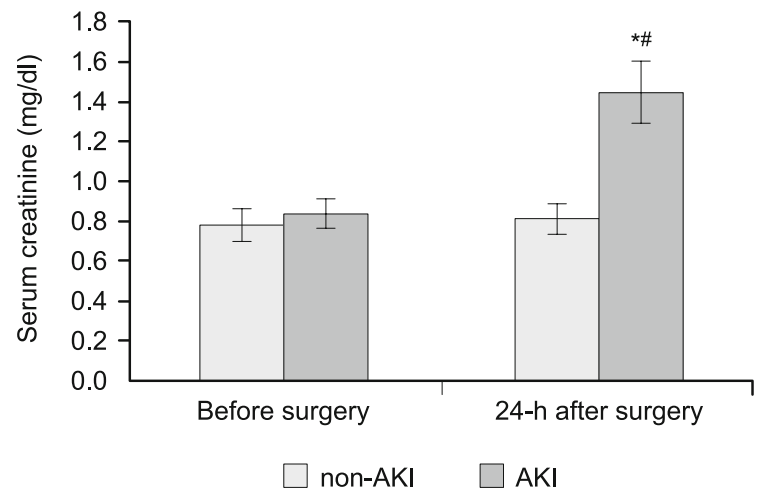

Fig. 1. Serum creatinine concentration in AKI and non-AKI groups. * $\mathbf{p}<0.01$ vs baseline values, and \# $\mathbf{p}<0.01$ vs non-AKI group. $\mathbf{n}=14$ in each group. Data was expressed as mean $\pm \mathrm{SD}$.

and between groups differences. Categorical variables with nominal scales were determined using chi-square or Fisher's exact tests. Correlations were analyzed by Pearson correlation coefficient test. The performance of miR-21 as a predictor for the diagnosis of AKI, was determined using the area under the curve (AUC) of the receiver operating characteristic (ROC) curve. The optimal cut-off points were selected as the values that minimized the geometric distance from both $100 \%$ sensitivity and $100 \%$ specificity on the ROC curves (27). p value less than 0.05 was considered significant.

\section{Results}

\section{Patients characteristics}

We included 14 (50\%) patients in AKI group and $14(50 \%)$ in non-AKI group. Among the study participants 15 (53.6\%) were male and $13(46.4 \%)$ were female. Also 15 (53.3 \%) had hypertension and $11(39.3 \%)$ had diabetes mellitus. Mean age of the study participants was $58.71 \pm 10.97$ years. Mean height, weight, and BMI were $162.04 \pm 9.32 \mathrm{~cm}, 62.75 \pm 14.25 \mathrm{~kg}$, and $23.83 \pm$ $4.45 \mathrm{~kg} / \mathrm{m}^{2}$, respectively. All CSA-AKI patients had a high level of sCr before their urinary output declined to the defined standard amount. At baseline, there was no significant difference in $\mathrm{sCr}$ between the two groups (Fig. 1). Table 1 summarizes demographic variables in two study groups.

\section{Serum concentration of miR-21 in AKI and non-AKI groups}

Figure 2 shows serum and urinary levels of miR-21 before and after surgery in AKI and non-AKI groups. Real-time PCR analysis demonstrated that baseline serum level of miR-21 in AKI group was significantly lower than non-AKI group (Fig. 2a) $(p=0.009)$.

As shown in Fig. 2a, in the AKI group, serum concentrations of miR-21 in 6,12 , and $24 \mathrm{~h}$ after surgery were decreased in comparison to those before surgery, but only this decrease was significant $6 \mathrm{~h}$ after surgery samples $(\mathrm{p}=0.008, \mathrm{p}=0.11$, and $\mathrm{p}$ $=0.19$, respectively).

There was no significant difference between the serum miR-21 levels before and after surgery in non-AKI group.

\section{Urine concentration of miR-21 in AKI and non-AKI groups}

As shown in Fig. 2b, baseline urine concentration of miR21 in AKI group was significantly lower than in non-AKI group $(p=0.003)$. while urinary levels of miR-21 in AKI group were markedly decreased 6,12 , and $24 \mathrm{~h}$ after surgery compared with those before surgery (Fig. 2b). These decrements were statistically significant 6 and $12 \mathrm{~h}$ after surgery samples $(\mathrm{p}=0.011, \mathrm{p}=$ 0.015 , and $p=0.056$ respectively). No significant difference was seen between the urinary miR-21 levels before and after surgery in non-AKI group (Fig. 2b).

Performance of plasma and urine miR-21 concentrations for detection of AKI

ROC curve analysis revealed that both postoperative serum and urine miR-21 concentrations can be used to detect AKI (Fig. 3). The area under the ROC curves values (AUC) were 0.81 (95\% CI: $0.65-0.97 ; p=0.004)$ for serum miR-21 ( $6 \mathrm{~h}$ after surgery), 0.90 (95\% CI: $0.79-0.99 ; \mathrm{p}=0.0001)$ for urine ( $6 \mathrm{~h}$ after surgery), and 0.86 (95\% CI: $0.71-0.98 ; \mathrm{p}=0.001)$ for urine ( $12 \mathrm{~h}$ after surgery). 

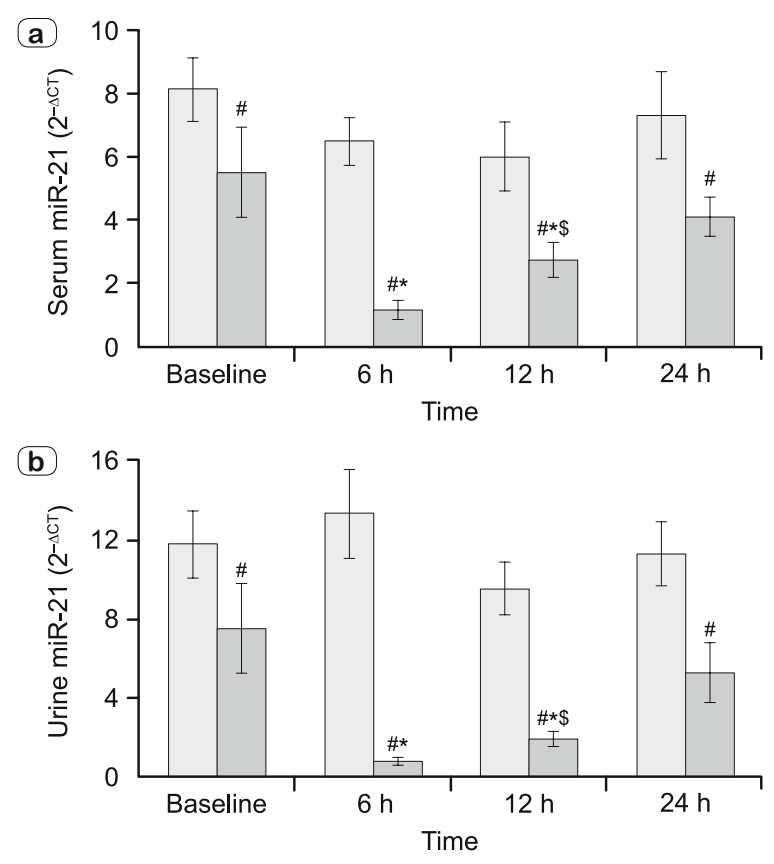

$\square$ non-AKI $\square$ AKI

Fig. 2. Serum (panel a) and urinary (panel b) miR-21 expression levels in AKI and non-AKI groups. Expression of miR-21 in each group $(n=14)$ was determined by real-time PCR in triplicate. ${ }^{*} \mathbf{p}<0.01$ vs baseline values, \# $p<0.01$ vs non-AKI group, and \$\# $p<0.05$ vs $6 \mathrm{~h}$ and $24 \mathrm{~h}$ sample values. Data were expressed as mean $\pm \mathrm{SD}$.

These data indicate that urine miR-21 concentrations especially 6 $\mathrm{h}$ after surgery is a more reliable marker than serum miR-21 for detection of established CSA-AKI.

The ROC curve analysis showed that neither preoperative serum miR-21 nor preoperative urine miR-21 can predict CSA-
AKI before surgery (AUC: $0.63,95 \%$ CI: $0.41-0.85 ; \mathrm{p}=0.12$; and AUC: 0.70, 95\% CI: 0.50-0.90; $\mathrm{p}=0.09$, respectively) (Fig. 3).

Correlation of miR-21 levels with age, ejection fraction, and duration of surgery and aorta clamp

Pearson correlation coefficient analysis identified no significant correlation between serum or urine miR-21 levels with patient's age, ejection fraction, duration of bypass and duration of aorta clamp.

\section{Discussion}

CSA-AKI is an important complication of major cardiac surgeries. The definition of CSA-AKI based on the decrease in GFR or increase in creatinine level is not applicable for early diagnosis of CSA-AKI $(6,31,32)$. These markers are useful $24 \mathrm{~h}$ after cardiac surgeries. More rapid diagnostic tests or diagnostic models are needed to prevent AKI and to detect high-risk patients (14,33-35). While resent studies have proposed the accuracy of miR-21 in prediction of CSA-AKI $(27,28)$, this accuracy to detect CSA-AKI during 24 $\mathrm{h}$ of postoperative period has been not fully investigated. The aim of this study was to test the hypothesis that miR-21 can detect CSAAKI within $24 \mathrm{~h}$ after surgery, and before reliable sCr increment.

The main findings of this study were as follows: firstly, patients with CSA-AKI had a lower serum and urinary levels of miR-21 than non-AKI developed patients; secondly, serum miR-21 expression level $6 \mathrm{~h}$ after cardiac surgery, and urinary miR-21 expression level 6 and $12 \mathrm{~h}$ after cardiac surgery were significantly lower in AKI group than in non-AKI group; thirdly, while both postoperative serum and urinary miR-21 levels can predict AKI development, urinary miR-21 was a better predictor than serum miR-21.

These findings are approximately in agreement with data published by Gaede et al who demonstrated a lower plasma level of miR-21 in patients with CSA-AKI before surgery (28). The
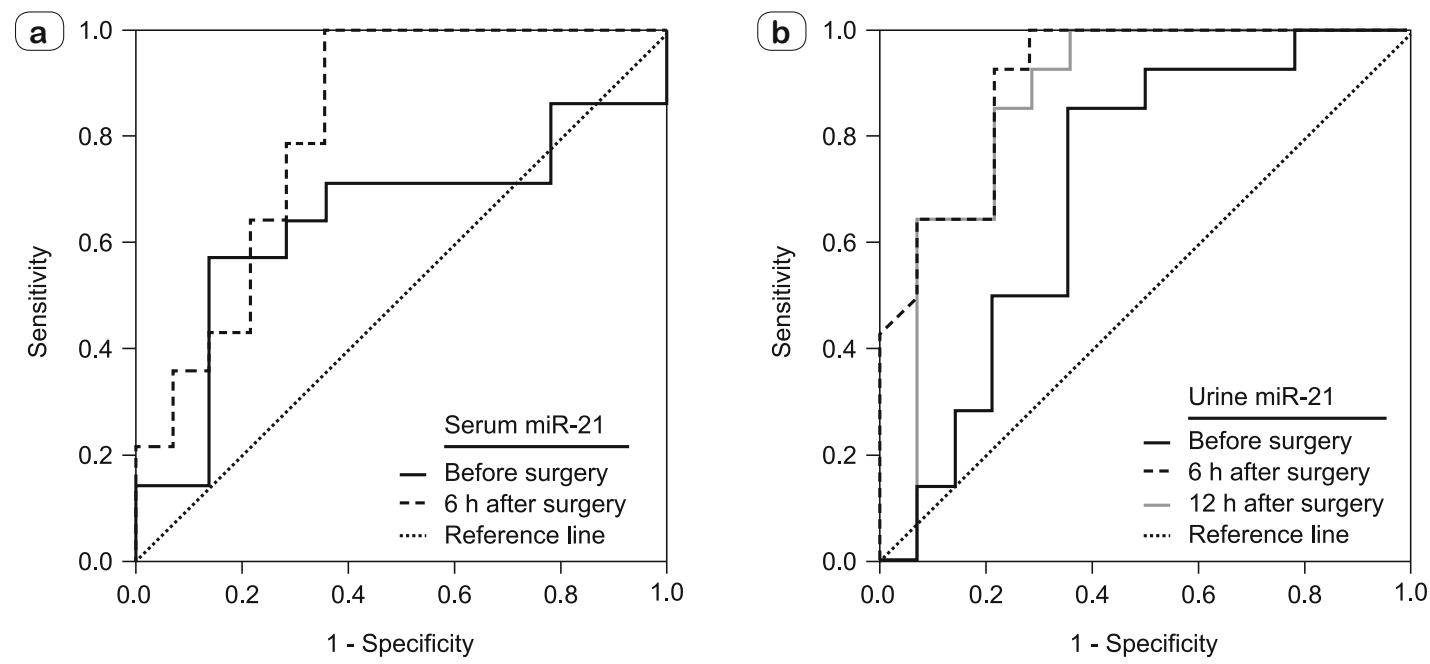

Fig. 3. Performance of urine (panel a) and serum (panel b) miR-21 for established CSA-AKI detection. Receiver-operating characteristic (ROC) curves show the distinctive performance of urine and serum miR-21 expression levels in AKI and non-AKI developed patients $(\mathrm{n}=14$ in each group). Data were expressed as mean $\pm \mathrm{SE}$. 
mentioned study was conducted on 115 patients, and CSA-AKI occurred in $36.5 \%$ of the patients. They measured plasma miR21 before and $4 \mathrm{~h}$ after cardiac surgery and showed that while plasma miR-21 level was lower in both samples of CSA-AKI patients compared with non-AKI patients, it was only significant before surgery (baseline) sample (28). Gaede et al also proposed that baseline level of miR-21 can be considered as a predictive biomarker for CSA-AKI detection.

Results of the present study show that the lowest level of serum and urinary miR-2 1 are recorded $6 \mathrm{~h}$ after surgery, and during the next hours after surgery miR-21 levels tend to return to their normal levels. Serum and urinary miR-21 at $12 \mathrm{~h}$ after surgery were significantly higher than those measured $6 \mathrm{~h}$ after surgery, as well as significantly lower than those measured at $24 \mathrm{~h}$ after surgery. These findings suggest that presumably miR-21 expression level is varied during the first $24 \mathrm{~h}$ in patients with CSA-AKI.

Theresults of our study are completely inconsistent with data published by Du etal who reported a higher level of plasma and urine miR21 in CSA-AKI patients $19.5 \mathrm{~h}$ after cardiac surgery (27). While they demonstrated that postoperative overexpression of miR-21 can be used as a predictor to identify AKI, no information was reported about preoperative changes of miR-21 between AKI and non-AKI groups.

Results of performed studies dealing with changes of miR-21 expression during the course of kidney injuries are incompatible. In the context of pathophysiological role for miR-21 in kidney disease progression, there are two controversial approaches. In one approach, most studies believe that miR-21 has pro-inflammatory and pro-apoptotic effects in kidney and its upregulation especially during ischemia-reperfusion induces cell injury and inhibits cell viability (27,36-38). In the other approach, several studies have suggested a reno-protective role for miR-21 and believe that upregulation of miR21 plays a physiological role in protecting cells from acute injury by antiapoptotic effects and silencing metabolic functions. However, in chronic conditions, long-term elevation of miR-21 is detrimental and promotes cells toward inflammation and fibrogenesis (39-43).

To the best of our knowledge this is the first data reporting postoperative decrement of miR-21 in CSA-AKI patients. However, our observation regarding preoperative decrement of miR-21 has been recently reported also by Gaede et al (28). It has been shown that miR-21 is highly expressed in the healthy kidney and can respond very rapidly to cell stress because it is already present within epithelial cells (43). It seems that that miR-21 is differentially expressed during different phases of renal injury. Our results suggest that presumably downregulation of miR-21 before and after cardiac surgery can make renal cells susceptible toward acute injury.

Studies on other biomarkers of AKI after major cardiac surgeries have shown that baseline renal function is an important factor in diagnostic accuracy of these biomarkers. Zhou et al have reported higher diagnostic accuracy for NGAL for diagnosis of CSA-AKI in individuals with normal baseline renal function (11). In this study, we divided the patients based on their renal function to AKI and non-AKI group. One strength of our study is the evaluation of the levels of miR-21 biomarkers in the groups of patients with and without normal renal function 6,12 and $24 \mathrm{~h}$ after cardiac surgery. This provides the possibility to compare the variations in miR-
21 level in both groups. Although the variations in the levels of miR-21 in both groups of AKI and non-AKI patients had similar patterns after 6 hour, 12 hours, and 24 hours after cardiac surgery, the changes were more prominent in CSA-AKI group. Therefore, it seems that this biomarker is more accurate in AKI group in our study. This fact is shown in both urine and serum levels of miR21 . This is incompatible with the results by Zhou et al (11). However, the fact that the study by Zhou et al is a meta-analysis on 4066 patients makes their results more reliable. Other fact is that NGAL and miR-21 are different biomarkers and their accuracy and the pattern of variations after cardiac surgeries are quite different.

Some recent available evidences from meta-analysis studies have reported successful usage of medications as prophylaxis of CSA-AKI in major cardiac surgeries. The mechanisms are poorly understood and may be related to increase in renal blood flow. Biomarkers like miR-21 which can predict CSA-AKI are ideal candidates for detecting the effectiveness of prophylaxis and understanding the respective mechanisms (23).

\section{Conclusion}

We aimed to test the hypothesis that miR-21 can detect CSAAKI in early hours after cardiac surgery. Based on obtained results, serum and urinary miR-21 expression levels $6 \mathrm{~h}$ after cardiac surgery were significantly lower in AKI group than in non-AKI group. Moreover, while both postoperative serum and urinary miR-21 levels can predict AKI development, urinary miR-21 was a better predictor than serum miR-21. More studies with higher sample sizes are recommended.

\section{References}

1. Hu J, Chen R, Liu S, Yu X, Zou J, Ding X. Global Incidence and Outcomes of Adult Patients With Acute Kidney Injury After Cardiac Surgery: A Systematic Review and Meta-Analysis. J Cardiothorac Vasc Anesth 2016; 30: $82-89$.

2. Corredor C, Thomson R, Al-Subaie N. Long-Term Consequences of Acute Kidney Injury After Cardiac Surgery: A Systematic Review and MetaAnalysis. J Cardiothorac Vasc Anesth 2016; 30: 69-75.

3. Xu JR, Zhu JM, Jiang J, Ding XQ, Fang Y, Shen B et al. Risk Factors for Long-Term Mortality and Progressive Chronic Kidney Disease Associated With Acute Kidney Injury After Cardiac Surgery. Medicine (Baltimore) 2015; 94: e2025.

4. O'Neal JB, Shaw AD, Billings FTt. Acute kidney injury following cardiac surgery: current understanding and future directions. Crit Care 2016; 20: 187.

5. Singh SP. Acute kidney injury after pediatric cardiac surgery. Ann Card Anaesth 2016; 19: 306-13.

6. Seelhammer TG, Maile MD, Heung M, Haft JW, Jewell ES, Engoren M. Kinetic estimated glomerular filtration rate and acute kidney injury in cardiac surgery patients. J Crit Care 2016; 31: 249-254.

7. Martensson J, Martling CR, Bell M. Novel biomarkers of acute kidney injury and failure: clinical applicability. Br J Anaesth 2012; 109: 843-850.

8. Mosa OF, Skitek M, Kalisnik JM, Jerin A. Evaluation of serum cysteinerich protein 61 and cystatin C levels for assessment of acute kidney injury after cardiac surgery. Ren Fail 2016; 38: 699-705. 
9. Wang X, Che M, Xie B, Xue S, Yan Y. Preoperative serum cystatin C combined with dipstick proteinuria predicts acute kidney injury after cardiac surgery. Ren Fail 2014; 36: 1497-1503.

10. Arthur JM, Hill EG, Alge JL, Lewis EC, Neely BA, Janech MG et al. Evaluation of 32 urine biomarkers to predict the progression of acute kidney injury after cardiac surgery. Kidney Int 2014; 85: 431-438.

11. Zhou F, Luo Q, Wang L, Han L. Diagnostic value of neutrophil gelatinase-associated lipocalin for early diagnosis of cardiac surgery-associated acute kidney injury: a meta-analysis. Eur J Cardiothorac Surg 2016; 49: 746-755.

12. Metzger J, Mullen W, Husi H, Stalmach A, Herget-Rosenthal S, Groesdonk HV et al. Acute kidney injury prediction in cardiac surgery patients by a urinary peptide pattern: a case-control validation study. Crit Care 2016; 20: 157.

13. Jung SY, Park JT, Kwon YE, Kim HW, Ryu GW, Lee SA et al. Preoperative Low Serum Bicarbonate Levels Predict Acute Kidney Injury After Cardiac Surgery. Medicine (Baltimore) 2016; 95: e3216.

14. Jorge-Monjas P, Bustamante-Munguira J, Lorenzo M, HerediaRodriguez M, Fierro I, Gomez-Sanchez E et al. Predicting cardiac surgery-associated acute kidney injury: The CRATE score. J Crit Care 2016; 31: 130-138.

15. Belley-Cote EP, Parikh CR, Shortt CR, Coca SG, Garg AX, Eikelboom JW et al. Association of cardiac biomarkers with acute kidney injury after cardiac surgery: A multicenter cohort study. J Thorac Cardiovasc Surg 2016; 152: 245-251 e4.

16. Ho J, Tangri N, Komenda P, Kaushal A, Sood M, Brar R et al. Urinary, Plasma, and Serum Biomarkers' Utility for Predicting Acute Kidney Injury Associated With Cardiac Surgery in Adults: A Meta-analysis. Am J Kidney Dis 2015; 66: 993-1005.

17. Saikumar J, Hoffmann D, Kim TM, Gonzalez VR, Zhang Q, Goering PL et al. Expression, circulation, and excretion profile of microRNA-21, -155, and -18a following acute kidney injury. Toxicol Sci 2012; 129: $256-267$.

18. Li YF, Jing Y, Hao J, Frankfort NC, Zhou X, Shen B et al. MicroRNA-21 in the pathogenesis of acute kidney injury. Protein Cell 2013; 4: 813-819.

19. Bonventre JV, Weinberg JM: Recent advances in the pathophysiology of ischemic acute renal failure. J Am Soc Nephrol 2003; 14: 2199-2210.

20. Morgan CJ, Gill PJ, Lam S, Joffe AR. Peri-operative interventions, but not inflammatory mediators, increase risk of acute kidney injury after cardiac surgery: a prospective cohort study. Intensive Care Med 2013; 39: 934-941.

21. Akcay A, Nguyen Q, Edelstein CL. Mediators of inflammation in acute kidney injury. Mediators Inflamm 2009; 2009: 137072.

22. Fujita S, Ito T, Mizutani T, Minoguchi S, Yamamichi N, Sakurai K, Iba H. miR-21 Gene expression triggered by AP-1 is sustained through a double-negative feedback mechanism. J Mol Biol 2008; 378: 492-504.

23. Zhou C, Gong J, Chen D, Wang W, Liu M, Liu B. Levosimendan for Prevention of Acute Kidney Injury After Cardiac Surgery: A Meta-analysis of Randomized Controlled Trials. Am J Kidney Dis 2016; 67: 408-416.

24. Xu J, Jiang W, Fang Y, Teng J, Ding X. Management of Cardiac Surgery-Associated Acute Kidney Injury. Contrib Nephrol 2016; 187: 131-142.

25. Shin SR, Kim WH, Kim DJ, Shin IW, Sohn JT. Prediction and Prevention of Acute Kidney Injury after Cardiac Surgery. Biomed Res Int 2016; 2016: 2985148.

26. McCullough PA, Bennett-Guerrero E, Chawla LS, Beaver T, Mehta RL, Molitoris BA et al. ABT-719 for the Prevention of Acute Kidney Injury in Patients Undergoing High-Risk Cardiac Surgery: A Randomized Phase 2b Clinical Trial. J Am Heart Assoc 2016; 5: e003549.
27. Du J, Cao X, Zou L, Chen Y, Guo J, Chen Z et al. MicroRNA-21 and risk of severe acute kidney injury and poor outcomes after adult cardiac surgery. PLoS One 2013; 8: e63390.

28. Gaede L, Liebetrau C, Blumenstein J, Troidl C, Dorr O, Kim WK et al. Plasma microRNA-21 for the early prediction of acute kidney injury in patients undergoing major cardiac surgery. Nephrol Dial Transplant 2016; 31: 760-766.

29. Yang XM, Tu GW, Gao J, Wang CS, Zhu DM, Shen B et al. A comparison of preemptive versus standard renal replacement therapy for acute kidney injury after cardiac surgery. J Surg Res 2016; 204: 205-212.

30. Khwaja A. KDIGO clinical practice guidelines for acute kidney injury. Nephron Clin Pract 2012; 120: c179-184.

31. Jefferies JL, Devarajan P. Early detection of acute kidney injury after pediatric cardiac surgery. Prog Pediatr Cardiol 2016; 41: 9-16.

32. Gavric A, Kalisnik JM. Novel biomarkers for early diagnosis of acute kidney injury after cardiac surgery in adults. Kardiochir Torakochirurgia Pol 2016; 13: 31-38.

33. Pannu N, Graham M, Klarenbach S, Meyer S, Kieser T, Hemmelgarn $\mathrm{B}$ et al. A new model to predict acute kidney injury requiring renal replacement therapy after cardiac surgery. CMAJ 2016; 188: 1076-1083.

34. Nah CW, Ti LK, Liu W, Ng RR, Shen L, Chew ST. A clinical score to predict acute kidney injury after cardiac surgery in a Southeast-Asian population. Interact Cardiovasc Thorac Surg 2016; 23: 757-761.

35. Jiang W, Teng J, Xu J, Shen B, Wang Y, Fang Y et al. Dynamic Predictive Scores for Cardiac Surgery-Associated Acute Kidney Injury. J Am Heart Assoc 2016; 5: e003754.

36. Lakhia R, Hajarnis S, Williams D, Aboudehen K, Yheskel M, Xing C et al. MicroRNA-21 Aggravates Cyst Growth in a Model of Polycystic Kidney Disease. J Am Soc Nephrol 2016; 27: 2319-2330.

37. Liu X, Hong Q, Wang Z, Yu Y, Zou X, Xu L. MiR-21 inhibits autophagy by targeting Rab11a in renal ischemia/reperfusion. Exp Cell Res 2015; 338: 64-69.

38. Zununi Vahed S, Omidi Y, Ardalan M, Samadi N. Dysregulation of urinary miR-21 and miR-200b associated with interstitial fibrosis and tubular atrophy (IFTA) in renal transplant recipients. Clin Biochem 2017; 50: 32-39.

39. Jiao $X$, Xu X, Teng J, Fang Y, Zhang $H$, Liang $M$ et al. miR-21 contributes to renal protection by targeting prolyl hydroxylase domain protein 2 in delayed ischemic preconditioning. Nephrology (Carlton) 2017; 22: 366-373.

40. Jia P, Teng J, Zou J, Fang Y, Wu X, Liang M et al. Xenon Protects Against Septic Acute Kidney Injury via miR-21 Target Signaling Pathway. Crit Care Med 2015; 43: e250-259.

41. Li Z, Deng X, Kang Z, Wang Y, Xia T, Ding N et al. Elevation of miR-21, through targeting MKK3, may be involved in ischemia pretreatment protection from ischemia-reperfusion induced kidney injury. J Nephrol 2016; 29: 27-36.

42. Xu X, Kriegel AJ, Jiao X, Liu H, Bai X, Olson J et al. miR-21 in ischemia/reperfusion injury: a double-edged sword? Physiol Genomics 2014; 46: 789-797.

43. Gomez IG, Nakagawa N, Duffield JS. MicroRNAs as novel therapeutic targets to treat kidney injury and fibrosis. Am J Physiol Renal Physiol 2016; 310: F931-944.

Received May 28, 2017. Accepted June 14, 2017. 